\title{
Significant response to lacosamide in a patient with severe chemotherapy-induced peripheral neuropathy
}

\author{
Samar A Ibrahim, MD; Zhanna Albany, MD; and Costantine Albany, MD
}

Division of Hematology and Medical Oncology, Department of Medicine, Indiana University Simon Cancer Center, Indianapolis, Indiana

\begin{abstract}
$\mathrm{C}$ hemotherapy-induced peripheral neuropathy (CIPN) is a major dose-limiting toxicity of potentially curative cancer therapy regimens. Cisplatin is the class of chemotherapy agent that has a broad spectrum of activity against several solid tumors, but it induces sensory neuropathy of upper and lower extremities. Cisplatin-induced peripheral neuropathy is usually in a "gloves and socks" distribution that can persist for months or years after completion of chemotherapy treatment. If the pain is severe, it affects the patient's long-term quality of life and can potentially result in chemotherapy dose reduction or treatment discontinuation. The mechanism of CIPN is not well understood, and a number of pathophysiological mechanisms have been proposed to explain the phenomenon. Although many therapies have been investigated for the prevention or treatment of CIPN, there is currently no accepted proven therapy. Here we report a case in which lacosamide alleviated painful CIPN symptoms. Lacosamide is an anticonvulsant drug that blocks the voltage-gated sodium channels in the neurons and may also be a promising novel candidate for the prevention and treatment of chemotherapy-induced peripheral neuropathy. Preclinical data support the role of lacosamide protective effect in a rat model of chemotherapy-induced neuropathy, randomized clinical trial is needed. ${ }^{1}$
\end{abstract}

\section{Case presentation}

Our patient was a 52-year-old white man with metastatic, high-grade urothelial carcinoma of the left renal pelvis. He had had a nephrectomy, followed by adjuvant chemotherapy MVAC (methotrexate, vincristine, adriamycin, and cisplatin) for 3 cycles. This treatment was complicated by painful peripheral neuropathy. The patient continued to complain of sharp pain, described as a pins-and-needles sensation in his hands and feet, as well as a loss of ability to recognize objects such as paper or money with his eyes closed.

His peripheral neuropathy was initially treated with gabapentin $400 \mathrm{mg}$ and morphine SR $30 \mathrm{mg}$, both twice a day, and oxycodone-acetaminophen $10-325 \mathrm{mg}$ as needed, but without much relief. When we saw him a year later for progression of the disease, we had planned to treat him with a cisplatin and gemcitabine regimen. However, we decided to try him on lacosamide because of his severe peripheral neuropathy. We had good experience using a sodium channel blocker to treat severe neuropathy using oxcarbazepine, but owing the many side effects and drug-drug interaction of oxcarbazepine we choose lacosamide because it is a safer and better-tolerated drug. The patient was started on lacosamide $100 \mathrm{mg}$ twice a day, and his pain immediately improved and he regained tactile sensation. He tolerated the drug very well and without side effects. Next, we initiated chemotherapy with cisplatin 70 $\mathrm{mg} / \mathrm{m}^{2}$ on Day 1 and gemcitabine $1,000 \mathrm{mg} / \mathrm{m}^{2}$ days 1 and 8 every 21 days, which he was able to tolerate well without worsening of peripheral neuropathy. However, he did develop nausea and vomiting from the chemotherapy regimen for few days and could not take his oral medications including lacosamide, which led to immediate worsening of his peripheral neuropathy symptoms. When the patient was able to restart lacosamide, his peripheral neuropathy symptoms resolved again and he continued to be free of peripheral neuropathy symptoms.

Accepted for publication February 23, 2015. Correspondence: Samar A Ibrahim, MD; samibrah@iupui.edu. Disclosures: The authors have no disclosures. JCSO 2015;13:202-204. @2015 Frontline Medical Communications. DOI 10.12788/ jeso.0136. 


\section{Discussion}

Lacosamide is a novel, sustained-release formulation of the functionalized amino acid, R-2-acetamido-N-benzyl-3methoxypropionamide. It modulates voltage-gated sodium channels by enhancing slow inactivating states without affecting the fast inactivation. ${ }^{2}$ Lacosamide affects neurons only, which depolarized or active for long period of time, which leads to inhibition of repetitive neuronal action potential and results in stabilization of hyperexitble membrane. ${ }^{2}$

The drug is rapidly absorbed from the gastrointestinal tract and has an oral bioavailability of nearly $100 \%$. In adults, it has low plasma protein binding, which reduces its potential interaction with other drugs. ${ }^{3}$ Beyreuther and colleagues found that lacosamide was effective as an analgesic in rat models for bone cancer pain and chemotherapy-induced neuropathic pain and even reduced hyperalgesia where morphine did not. ${ }^{4}$ It has also been found to attenuate thermal and tactile allodynia and mechanical hyperalgesia in a vincristine-induced neuropathy in rats and to show a protective effect in a rat model of vincristine neuropathy. ${ }^{1}$ The drug has shown showed efficacy in several short- and long-term phase 2 clinical trials in humans with diabetic neuropathic pain. ${ }^{5,6}$ It is used at doses of $200 \mathrm{mg}, 400 \mathrm{mg}$, or $600 \mathrm{mg}$ daily, given as a divided dose. Lacosamide is generally well tolerated in adult patients in doses up to $400 \mathrm{mg} /$ day. The most common side effects are headache, dizziness, and nausea. Less common side-effects are diplopia, blurred vision, forgetfulness, discouragement, and lack of appetite. ${ }^{7}$

CIPN is a common and often debilitating dose-limiting side effect associated with several commonly used chemotherapeutic agents, including platinum, taxanes, and vinca alkaloids. ${ }^{8}$ CIPN occurs in $30 \%-40 \%$ of patients, but its incidence may be as high as $70 \% .^{9}$ The symptoms most commonly associated with it are sensory neuropathies, including paresthesia, allodynia, dysthesia, and pain, which is burning, or electric shock like. ${ }^{10}$ The symptoms often begin in the fingers and toes and spread proximally in a "glove and socks" distribution. ${ }^{11}$ CIPN may begin any time from weeks to months after treatment initiation and reaches a peak at or after the end of treatment. The symptoms get progressively worse over the course of the chemotherapy.

The exact mechanism of CIPN has not been fully described and can differ within classes of chemotherapeutic agents. In most cases, CIPN is only partially reversible and can be permanent. Painful symptoms may persist 2-6 months beyond discontinuation of chemotherapy, a phenomenon known as "coasting." 12 In addition, patients who have had evidence of CIPN, even if signs and symptoms improved significantly after discontinuation of the chemotherapy, are at increased risk of progressive neuropathic symptoms if they are exposed to other neurotoxic agents. This may influence the choice of future chemotherapeutic agents. As such, CIPN may result in chemotherapy dose reduction, the selection of less efficacious therapies, or even cessation of cancer treatment, all of which can be detrimental. ${ }^{13}$ Therefore, it is of great importance to find effective treatment for CIPN in cancer patients.

Findings from randomized, controlled trials with a variety of drugs with diverse mechanisms of action have shown there to be no effective treatment for CIPN, with the exception of duloxetine. ${ }^{14}$ Few agents - including topical baclofen-amitriptyline-ketamine gel, ${ }^{15}$ venlafaxine, ${ }^{16}$ and pregabalin ${ }^{17}$ - have preliminary data to support their use in the treatment of CIPN. It is important to undertake further investigation into the natural history of CIPN associated with various classes of neurotoxic chemotherapy agents. This may provide important insight for the development of new strategies for the prevention and treatment of CIPN.

\section{Conclusion}

Lacosamide may be a promising novel candidate for the prevention and treatment of cancer pain including chemotherapy-induced peripheral neuropathy. Here we report on a successful case of cisplatin-induced neuropathy treated with lacosamide. As far as we know, this is the first reported case of its kind. There should be further investigation into the natural history of CIPN associated with various classes of neurotoxic chemotherapy agents. This may provide important insight for the development of new strategies in prevention and treatment of CIPN.

\section{References}

1. Geis C, Beyreuther BK, Stöhr T, Sommer C. Lacosamide has protective disease modifying properties in experimental vincristine neuropathy. Neuropharmacology. 2011;61:600-607.

2. Errington AC, Stöhr T, Heers C, Lees G. The investigational anticonvulsant lacosamide selectively enhances slow inactivation of voltage-gated sodium channels. Mol Pharmacol. 2008;73:157-169.

3. Cawello W, Bökens H, Nickel B, Andreas JO, Halabi A. Tolerability, pharmacokinetics, and bioequivalence of the tablet and syrup formulations of lacosamide in plasma, saliva, and urine: saliva as a surrogate of pharmacokinetics in the central compartment. Epilepsia. 2013;54:81-88.

4. Beyreuther BK, Callizot N, Brot MD, Feldman R, Bain SC, Stöhr T. Antinociceptive efficacy of lacosamide in rat models for tumor- and chemotherapy-induced cancer pain. Eur J Pharmacol. 2007;565:98104.

5. Wymer JP, Simpson J, Sen D, Bongardt S; Lacosamide SP742 Study Group. Efficacy and safety of lacosamide in diabetic neuropathic pain: an 18-week double-blind placebo-controlled trial of fixed-dose regimens. Clin J Pain. 2009;25:376-385.

6. Rauck RL, Shaibani A, Biton V, Simpson J, Koch B. Lacosamide in painful diabetic peripheral neuropathy: a phase 2 double-blind placebo-controlled study. Clin J Pain. 2007;23:150-158.

7. Chung S, Ben-Menachem E, Sperling MR, et al. Examining the clinical utility of lacosamide: pooled analyses of three phase II/III clinical trials. CNS Drugs, 2010;24:1041-1054.

8. Wolf S, Barton D, Kottschade L, Grothey A, Loprinzi C. Chemotherapy-induced peripheral neuropathy: prevention and treatment 


\section{Case Report}

strategies. Eur J Cancer. 2008;44:1507-1515.

9. Hershman DL, Lacchetti C, Dworkin RH, et al. Prevention and management of chemotherapy-induced peripheral neuropathy in survivors of adult cancers: American Aociety of Clinical Oncology clinical practice guideline. J Clin Oncol. 2014;32:1941-1967.

10. Wolf SL, Barton DL, Qin R, et al. The relationship between numbness, tingling, and shooting/burning pain in patients with chemotherapy-induced peripheral neuropathy (CIPN) as measured by the EORTC QLQ-CIPN20 instrument, N06CA. Support Care Cancer. 2012;20:625-632.

11. Stubblefield MD, Burstein JF, Burton AW, et al. NCCN task force report: management of neuropathy in cancer. J Natl Compr Canc Netw. 2009;7 Suppl 5:S1-S26; quiz S27-S28.

12. Argyriou AA, Bruna J, Marmiroli P, Cavaletti G. Chemotherapyinduced peripheral neurotoxicity (CIPN): an update. Crit Rev Oncol Hematol. 2012;82:51-77.

13. Pachman DR, Barton DL, Watson JC, Loprinzi CL. Chemothera- py-induced peripheral neuropathy: prevention and treatment. Clin Pharmacol Ther. 2011;90:377-387.

14. Smith EM, Pang H, Cirrincione C, et al; Alliance for Clinical Trials in Oncology. Effect of duloxetine on pain, function, and quality of life among patients with chemotherapy-induced painful peripheral neuropathy: a randomized clinical trial. JAMA. 2013;309:1359-1367.

15. Barton DL, Wos EJ, Qin R, et al. A double-blind, placebo-controlled trial of a topical treatment for chemotherapy-induced peripheral neuropathy: NCCTG trial N06CA. Support Care Cancer. 2011;19:833-841.

16. Durand JP, Deplanque G, Montheil V, et al. Efficacy of venlafaxine for the prevention and relief of oxaliplatin-induced acute neurotoxicity: results of EFFOX, a randomized, double-blind, placebo-controlled phase III trial. Ann Oncol. 2012;23:200-205.

17. Saif MW, Syrigos K, Kaley K, Isufi I. Role of pregabalin in treatment of oxaliplatin-induced sensory neuropathy. Anticancer Res, 2010;30:2927-2933. 